\title{
Diagnostic Accuracy of Bone Scintigraphy in the Early Prediction of MRONJ
}

\author{
Federica Rocchetti ${ }^{1}$, Marco Giulietti ${ }^{1}$, Daniele Pergolini ${ }^{1}$, Gaspare Palaia ${ }^{1}$, Domenico Gaglioti, Umberto Romeo ${ }^{1}$, \\ Alessandro Del Vecchio ${ }^{1}$
}

1 Sapienza University of Rome

Funding: The author(s) received no specific funding for this work.

Potential competing interests: The author(s) declared that no potential competing interests exist.

\section{Abstract}

Since Medication-Related Osteonecrosis of the Jaw (MRONJ) is challenging to treat, prevention and early detection are fundamental to limit progression. While some imaging techniques such as computed tomography (CT) scans are helpful to estimate the extent of osteonecrosis in clinically evident MRONJ, methods for early detection and identification of patients at risk for MRONJ need further investigations. The aim of our retrospective study was to evaluate the role of bone scintigraphy (BS) in the early prediction of MRONJ. BS of patients treated with antiangiogenic or antiresorptive therapy for bone metastases were evaluated for pathological tracer uptake of the jaws. Sensitivity, specificity, predictive values of BS for the prediction of MRONJ were investigated. Moreover, the latency from the first presence of pathological tracer uptake in BS to clinically evident MRONJ was determined. The significance of pathologic BS for MRONJ detection was calculated by Fisher's exact test and the odds ratio was determined. A total of 19 patients with representative BS were recruited from our Department database. Whole body BS in anterior and posterior positions were reviewed by a specialist in nuclear medicine, blinded to MRONJ diagnosis, for pathological tracer uptake of the jaws. Results were compared to development of clinically evident MRONJ. Sensitivity and specificity of BS for MRONJ prediction were respectively $90 \%$ and $82 \%$. Positive and negative predictive values were $64 \%$ and $96 \%$, respectively. Median time from the first presence of pathological tracer uptake in BS to clinically evident MRONJ was 20.3 months (range 7-41). Pathologic findings on BS were significantly $(p<0.001)$ more often observed in patients who developed MRONJ compared to patients who did not. The risk to develop MRONJ was 41.4-fold increased when BS was pathologic. According to our results, BS is an accurate method for the prediction of MRONJ.

\section{Introduction}

Since Medication-Related Osteonecrosis of the Jaw (MRONJ) is challenging to treat and can have a significant negative impact on quality of life of the patients, prevention and early detection are fundamental to limit progression. ${ }^{[1][2]}$ While some imaging techniques like computed tomography (CT) scans and Magnetic Resonance Imaging (MRI) are helpful to estimate the extent of osteonecrosis in clinically evident MRONJ, there is no convincing data available if any techniques improve early detection of MRONJ. ${ }^{[3]}$ Bone scintigraphy (BS) continues to be the most widely used technique for the 
investigation of skeletal metastasis primarily due to its widespread availability. BS can detect metabolic, vascular, and pathophysiologic changes in bone earlier than CT scan and MRI. The aim of our study was to evaluate the diagnostic accuracy of BS in the early prediction of MRONJ.

\section{Materials and Methods}

To address the specific study aim, a retrospective cohort study design was used. Written informed consent was not deemed necessary according to national medical ethical guidelines due to the retrospective nature of the study. The study was approved by our institutional review board. The following question was structured according to the PICO (patient, intervention, comparison, and outcome) study design:

Is BS effective for the early prediction of MRONJ?

The cohort was composed of patients treated with antiangiogenic or antiresorptive therapy for skeletal metastases and who underwent to multiple BS to monitor the metastases referred to the Department of Oral and Maxillofacial Sciences, Sapienza University of Rome from January 2019 to January 2020 for the odontostomatologic management. Exclusion criteria consisted of patients with insufficient follow-up to evaluate MRONJ development. Data were collected on demographics and MRONJ characteristics, type and duration of therapy and eventually adjunctive therapy. Previous whole body Technetium-99m hydroxymethylene diphosphonate (Tc99m-Hmdp) bone scans in anterior and posterior views of all patients were reviewed by a specialist in nuclear medicine with 15 years of experience, blinded to MRONJ presentation, for pathological tracer uptake of the maxilla and mandible. Visual interpretation were performed and the findings were related to the presence of clinically evident MRONJ staged by the SICMF-SIPMO classification system. Statistical analysis was conducted using the R statistical package (version 3.6.1; R Foundation for Statistical Computing, Vienna, Austria). Sensitivity, specificity, positive and negative predictive values of BS for the early detection of MRONJ were investigated. Moreover, the latency from the first presence of pathological tracer uptake in BS to clinically evident MRONJ was determined. The significance of pathologic BS for MRONJ detection was calculated by Fisher's exact test and the odds ratio determined. A p-value less than 0.05 was considered statistically significant.

\section{Results}

We identified 19 patients ( 12 females and 7 males) with mean age of $65.1 \pm 12.3$ years old. Breast cancer was the primary malignancy $(61.1 \%)$, followed by prostate cancer $(16,6 \%)$, kidney cancer $(11,1 \%)$ and lung cancer $(11,1 \%)$. Ten (52.6\%) patients were medicated with zoledronate and nine (47.4\%) with denosumab. The route of administration was intravenous for all patients. Mean duration of medication was $44.0 \pm 30.0$ months. A total of 45 representative BS were reviewed. Twenty-eight of 76 quadrants evaluated presented pathological uptake and among these 18 developed MRONJ (Figure 1). 


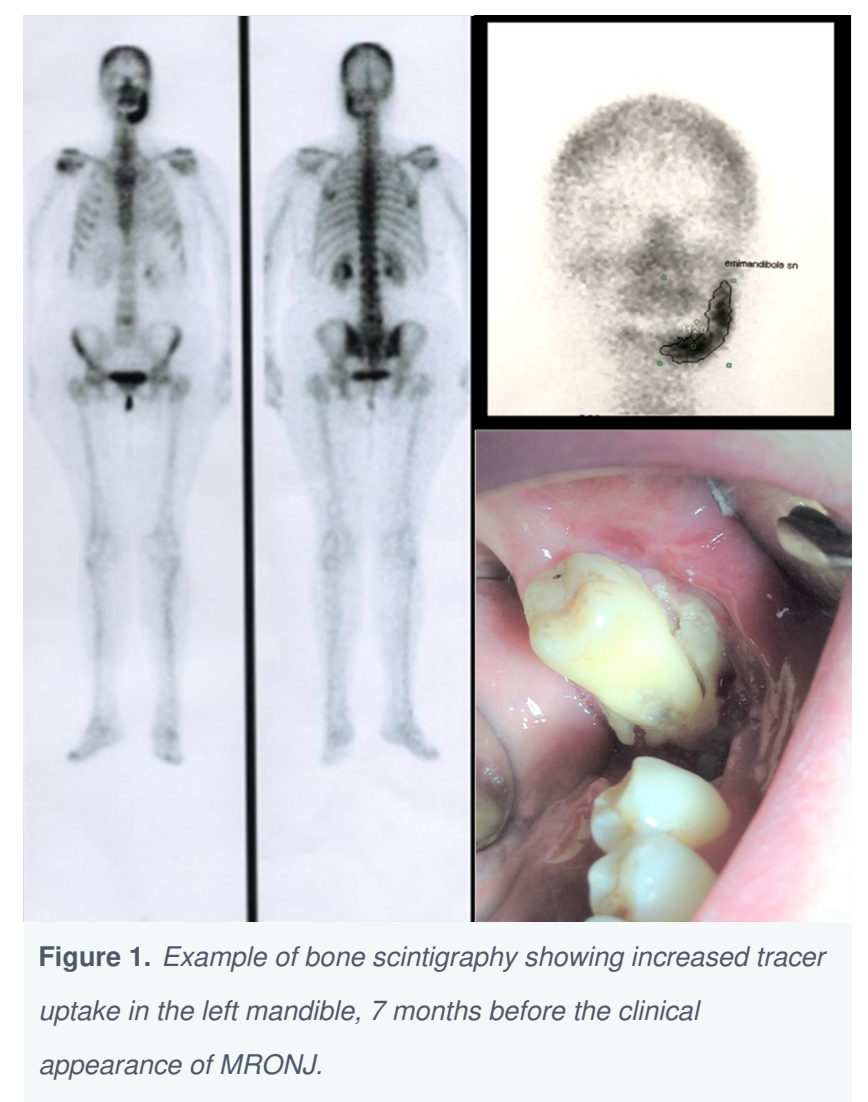

Out of 48 quadrants without tracer accumulation, 2 quadrants developed MRONJ.

Sensitivity and specificity of BS for MRONJ prediction were respectively $90 \%$ and $82 \%$. Positive and negative predictive values were 64 and $96 \%$, respectively. Median time from the first pathological tracer uptake in BS to clinical evident MRONJ was 20.3 months (range 7-41). Pathologic findings on BS were significantly $(p<0.001)$ more often observed in patients who developed MRONJ compared to patients who did not. The risk to develop a clinically significant MRONJ was 41.4-fold increased when BS was pathologic.

\section{Conclusions}

According to our results, BS with pathological tracer uptake in the maxillo-facial region is associated with a markedly increased risk to develop clinically evident MRONJ. Conversely, if BS is negative, development of MRONJ in the future is unlikely. For daily clinical practice of patients receiving antiangiogenic or antiresorptive therapy, we recommend not only to assess tumour stage and treatment response but also to check for pathological tracer uptake in the jaws when BS has been performed. The oral healthcare providers will accurately monitor the area of BS with high tracer uptake in order to eliminate the presence of risk factors and prevent the development of MRONJ. 


\section{References}

1. 'Campisi et al.. (2020). . Medication-Related Osteonecrosis of Jaws (MRONJ) Prevention and Diagnosis: Italian Consensus Update 2020.. Int J Environ Res Public Health.

2. 'Gianluca Tenore, Ahmed Mohsen, Antonella Francesca Rossi, Gaspare Palaia, et al. (2020).Does MedicationRelated Osteonecrosis of the Jaw Influence the Quality of Life of Cancer Patients? Biomedicines, vol. 8 (4), 95. doi:10.3390/biomedicines8040095.

3. ^Akira Baba, Tazuko K Goto, Hiroya Ojiri, Mutsumi Takagiwa, et al. (2018).CT Imaging Features of Antiresorptive agent-Related Osteonecrosis of the Jaw/Medication-Related Osteonecrosis of the Jaws. Dentomaxillofacial Radiology. doi:10.1259/dmfr.20170323. 